\title{
Antimicrobial dependence of silver nanoparticles on surface plasmon resonance bands against Escherichia coli
}

\author{
Nichrous G Mlalila',2 \\ Hulda Shaidi Swai' \\ Askwar Hilonga ${ }^{3}$ \\ Dattatreya M Kadam²
}

'School of Life Sciences and Bioengineering, Nelson Mandela African Institution of Science and Technology, Arusha, Tanzania; ${ }^{2}$ ICARCentral Institute of Post-Harvest Engineering and Technology (ICARCIPHET), Ludhiana, Punjab, India; ${ }^{3}$ Department of Materials Science and Engineering, Nelson Mandela African Institution of Science and Technology, Arusha, Tanzania
Correspondence: Nichrous Mlalila School of Life Sciences and Bioengineering, Nelson Mandela African Institution of Science and Technology, PO Box 447, Arusha, Tanzania Tel +25576277 1747

Email nichogm_2006@yahoo.com/ mlalilan@nm-aist.ac.tz
This article was published in the following Dove Press journal:

Nanotechnology, Science and Applications

20 December 2016

Number of times this article has been viewed

\begin{abstract}
This study presents a simple and trouble-free method for determining the antimicrobial properties of silver nanoparticles (AgNPs) based on the surface plasmon resonance (SPR) bands. AgNPs were prepared by chemical reduction method using silver nitrates as a metallic precursor and formaldehyde $(\mathrm{HCHO})$ as a reducing agent and capped by polyethylene glycol. Effects of several processing variables on the size and shape of AgNPs were monitored using an ultraviolet-visible spectrophotometer based on their SPR bands. The formed particles showing various particle shapes and full width at half maximum (FWHM) were tested against Escherichia coli by surface spreading using agar plates containing equal amounts of selected AgNPs samples. The NPs exhibited higher antimicrobial properties; however, monodispersed spherical NPs with narrow FWHM were more effective against $E$. coli growth. The NPs prepared are promising candidates in diverse applications such as antimicrobial agents in the food and biomedical industries.
\end{abstract}

Keywords: antimicrobial agent, bandwidth, full width at half maximum, nanoparticles, particle size

\section{Introduction}

The application of nanotechnology in various fields and technologies is increasing rapidly. In the field, the application of metallic nanoparticles (NPs) in the technological sector has gained special attention in many areas such as photonic, biosensing, nanosensors, catalytic, cell electrodes, optics, and antimicrobial activities ${ }^{1-3}$ owing to their unique optical, physical, and chemical properties. ${ }^{4,5}$ Recently, comprehensive studies have been conducted to characterize many nanometals for industrial applications. Silver NPs (AgNPs) have gained potential application in most nanomaterial-based consumer products in the market. ${ }^{6,7}$ AgNPs have gained application in biological sciences, food science, pharmaceuticals, packaging, electronic systems, mechanics, and information technology, among others. ${ }^{8-12}$ However, these applications are based on properties that are defined by their shape, size, configuration, and crystal orientations. ${ }^{13,14} \mathrm{AgNPs}$ have a high surface-to-volume ratio ${ }^{15}$ with unique properties for novel applications, on the basis of which many strategies have been developed to control the shapes, sizes, and orientations of AgNPs for particular industrial applications. ${ }^{5,16}$

One of the attractive applications of AgNPs in the food and biomedical industries is their antimicrobial properties. It has been reported that AgNPs have antimicrobial activity against 650 strains of spoilage and pathogenic microorganisms ranging from bacteria, fungi, viruses, molds, and yeasts. ${ }^{15,17}$ They can exhibit the antimicrobial nature even in concentrations as low as 10 ppm. ${ }^{2,18,19}$ Their broad-spectrum killing nature submit your manuscript Dovepress if in $>$ 
has been reported to be associated with their diverse and oligodynamic antimicrobial mechanisms. ${ }^{6}$ The antimicrobial activity of AgNPs works through the following mechanism: 1) blocking the active respiratory chains of organisms, 2) disrupting the cellular membrane leading to leakage of cellular contents, 3) binding to the functional groups of microbial proteins that lead to protein denaturation and DNA malfunctions, and 4) blocking nutrient transportation enzymes across the cell membrane. ${ }^{1,15,19-25}$ This precludes the possibility of microorganisms developing resistance genes against AgNPs' antimicrobial activity. ${ }^{2,13,14,23}$

As a result, silver in the nano size range has emerged as the most exploited metallic nano-antimicrobial for industrial applications, with as many as 313 of 565 nanomaterialbased consumer products in the market reported to contain AgNPs. ${ }^{6,7}$ In this regard, AgNPs have been reported by several authors to be the most effective antimicrobial agent of choice against the development of antibiotics-resistant strains of microorganisms. Recently, synergetic antibiotics of chloramphenicol, erythromycin, penicillin G, ampicillin, kanamycin, amoxicillin, clindamycin, and vancomycin containing AgNPs have been reported., ${ }^{3,26}$ This synergism has improved antimicrobial effects against microbial strains including Staphylococcus aureus, Micrococcus luteus, Salmonella typhi, and Escherichia coli compared to the activity of these antibiotics alone. In addition, Liu et $\mathrm{al}^{27}$ developed modified silver nanorods with polyvinylpyrrolidone-polyethylene glycol (PEG) as a candidate for adjuvant human immunodeficiency virus vaccine delivery owing to its safety and low toxicity in biological systems compared to silver nitrates, which exhibit toxicity at $2 \mu \mathrm{g} / \mathrm{mL} .{ }^{8,28}$ Erick and Padmanabhan ${ }^{29}$ showed that AgNPs synthesized by green methods have exhibited high larvicidal and pupicidal activity against the malarial vector Anopheles stephensi.

The antimicrobial activities of AgNPs have been reported mainly as the factor of concentration of NPs in the media, shapes, and sizes of NPs. ${ }^{6}$ Therefore, much painstaking work has, over the decades, sought to optimize several factors that affect the shapes and sizes of NPs. The most notable studies have centered on the effects of shapes and sizes on the antimicrobial nature of NPs. ${ }^{13,14,24,30-32}$ The debate is currently over whether antimicrobial properties of AgNPs depend on the shape or size of NPs. For the successful production of certain characteristics of AgNPs, several factors have been considered, including the reaction time, mixing rates, the order of mixing, the concentration and volume of reacting species, and $\mathrm{pH}$ of media. ${ }^{33}$ These can affect the shape, size, stability, $\mathrm{pH}$, rheology, crystallinity, structures, and nucleation growth of NPs. However, to date, no research has reported on the association of antimicrobial activity of AgNPs with the symmetrical phenomena of surface plasmon resonance (SPR) bands associated with their full width at half maximum (FWHM) or bandwidths as reported from ultraviolet-visible (UV-Vis) spectrophotometer bands.

The extinction peaks and bandwidths depict many features of metallic NPs beyond spectra positions. The size, shape, homogeneity, and dispersity of the AgNPs from the SRP and bandwidth can be well reported and give their relationship to the antimicrobial nature. Spherical-shaped NPs have been reported to exhibit the highest extinctions peaks compared to large NPs, which shift the absorption to higher absorption wavelengths. ${ }^{13,23}$ The presence of different shapes in the produced NPs shows more than two peaks in the spectrum, leading to high distribution of particle size (PS). In this study, the chemical reduction method was used for the synthesis of AgNPs, employing silver nitrate as metallic precursor, formaldehyde ( $\mathrm{HCHO}$ ) solution as reducing agent, PEG as capping material, and sodium hydroxide $(\mathrm{NaOH})$ as the reaction promoter. Chemical reduction is the most attractive method in view of simplicity, process control, stable products, and affordable matrix-solvent purification process, possible on surface modifications, scale-up production, and high homogeneity of NPs. The focus was to understand the antimicrobial activity of AgNPs based on the SPR bands, representing the shapes and dispersity of NPs as explored from reaction time and concentration of $\mathrm{PEG}$. The processing was subjected to various formulation parameters to influence the reduction of NPs of different symmetrical distributions from $100 \mathrm{~nm}$ to $30 \mathrm{~nm}$.

\section{Experimental approach Materials and reagents}

All chemicals used in the experiment were analytic reagents. Silver nitrate $\left(\mathrm{AgNO}_{3}\right)$ content $99.9 \%$ (Central Drug House (P) Ltd, New Delhi, India), PEG (MW 6,000-7,500; Sisco Research Laboratories Pvt. Ltd, Mumbai, India), and HCHO solution $(37 \%-41 \% \mathrm{w} / \mathrm{v})$ and $\mathrm{NaOH}$ pellets $(98.0 \%)$ (SDFCL, Mumbai, India) were the reagents that were used directly without further purification.

\section{Preparation of AgNPs}

Colloidal silver NPs were synthesized by the chemical reduction of $\mathrm{AgNO}_{3}$ in the presence of $\mathrm{HCHO}$ as reducing agent; PEG and $\mathrm{NaOH}$ were used as capping agent and reduction catalyst, respectively. In all experiments, constant amounts of $1 \mathrm{mM} \mathrm{AgNO}_{3}$ were used and mixed with constant molar 
ratios of $\mathrm{HCHO}$ or $\mathrm{NaOH}$ solutions to $\mathrm{AgNO}_{3}$ at ten factors. Then, PEG was added into the reaction beaker to stabilize the reaction process. The volumes of the respective concentrations of PEG used for capping the formed AgNPs were $11,15,20,25$, and $30 \mathrm{~mL}$, and $\mathrm{NaOH}$ solution was added to the reaction system to initiate the reduction as well as to achieve a reaction time of several minutes. All measurements were performed at room temperature $\left(25^{\circ} \mathrm{C}\right)$. Magnetic stirring was applied throughout the entire synthesis. The reaction time for other control experiments is $30 \mathrm{~min}$ if not mentioned otherwise.

After the completion of the reaction, the NP suspensions were allowed to cool at room temperature and mixed with a certain amount of acetone to allow for the generation of brown precipitate of AgNPs. To remove the unreacted $\mathrm{Ag}^{+}$ ions and the $\mathrm{HCHO}$, protected silver colloids were separated from the solution by first adding acetone of about five times the total solution volume and then centrifuging at 12,000 rpm for $20 \mathrm{~min}$. The black gel-like material obtained was then washed at least three times by acetone to remove as much $\mathrm{HCHO}$ as possible. The product was dried at $40^{\circ} \mathrm{C}$ for $10 \mathrm{~h}$ in a vacuum dryer and could be redispersed in either ethanol or water for further uses.

\section{Characterizations Dynamic light scattering (DLS)}

The PS distribution and zeta potential of the AgNPs were measured using DLS (Zetasizer Nano ZS; Malvern Instruments, Malvern, UK). DLS data were analyzed at $25^{\circ} \mathrm{C}$ and with a fixed light incidence angle of detection of $173^{\circ}$ using the backscattering technique in optically homogeneous square polystyrene cells. The mean hydrodynamic diameter (PS, Z-average) and polydispersity index (PDI) of the analyzed samples were obtained by calculating the average of 14 runs. All the presented results of the PSs are listed as average values from three independent measurements.

\section{UV-Vis spectrophotometer}

The UV-Vis spectra of the silver dispersion during the reduction process were monitored using a LABINDIA UV (UV-VIS Spectrophotometer 3000+; Labindia Analytical Instruments Pvt. Ltd, Hyderabad, India).

\section{Antimicrobial studies of AgNPs}

The antimicrobial efficacies of the prepared AgNPs were tested against $E$. coli. The slant of preserved E. coli was inoculated in $9 \mathrm{~mL}$ of agar broth and incubated for $24 \mathrm{~h}$ at $37^{\circ} \mathrm{C}$ and diluted to $10^{5}$ colony forming units $/ \mathrm{mL}$ in peptone buffer. Then, $100 \mathrm{~mL}$ of inoculums were inoculated onto the surface of prepared tryptone soya agar plates containing the same $5 \mu \mathrm{g} / \mathrm{mL}$ of AgNPs of the selected samples with the respective positive control of $\mathrm{HCHO}$ solution in the Petri dish. The surface spread plates were incubated at $37^{\circ} \mathrm{C}$ for $24 \mathrm{~h}$ in triplicates. The enumeration of viable cells of $E$. coli in the incubated plates was taken after $24 \mathrm{~h}$ of incubation to observe the efficacy of the NPs based on SPR and bandwidths. The results were expressed as colony forming unit $/ \mathrm{mL}$ from both conventional and control plates.

\section{Results and discussion}

Two main reaction phases mark the formation of AgNPs: nucleation growth, which begins the formation of NPs, and then the coagulation and coalescence processes. Both formulation - such as concentration, dilution, compositions - and process parameters - such as temperature, time, pressure, mixing procedure - can be used to control the growth of the prepared NPs. In this case, several optimization strategies were used to reach the conclusions based on the results presented in what follows. The results presented are based on dropwise addition of silver nitrate solution in the mixture of PEG and HCHO solution and then the addition of $\mathrm{NaOH}$ solution.

\section{Theoretical formation of $\mathrm{AgNO}_{3}$ from $\mathrm{HCHO}$ reduction}

The reduction phenomena of $\mathrm{AgNO}_{3}$ to $\mathrm{AgNPs}$ using $\mathrm{HCHO}$ have been reported extensively during the last decade. ${ }^{34-38}$ However, two main synthetic routes of AgNPs can be developed when using $\mathrm{HCHO}$ as reducing agent. The first is that the presence of $\mathrm{NaOH}$ in the reaction leads to the Cannizzaro reaction, which is essentially the auto-oxidation-reduction of $\mathrm{HCHO}$. Consequently, formic acid $(\mathrm{HCOOH})$ and methanol $\left(\mathrm{CH}_{3} \mathrm{OH}\right)$ are formed, whereby $\mathrm{CH}_{3} \mathrm{OH}$ is easily converted into $\mathrm{HCOOH}$. The $\mathrm{HCOOH}$ is produced as the sodium salt, sodium formate ( $\mathrm{HCOONa}$ ). HCHO undergoes the Cannizzaro reaction as follows:

$$
\begin{gathered}
2 \mathrm{HCHO}+\mathrm{OH}^{-} \rightarrow \mathrm{HCOO}^{-}+\mathrm{CH}_{3} \mathrm{OH} \\
\mathrm{Ag}^{+}+\mathrm{HCOO}^{-} \rightarrow \mathrm{Ag}+\mathrm{CO}_{2}+\frac{1}{2} \mathrm{H}_{2}
\end{gathered}
$$

However, depending on the reaction sequence such as mixing of $\mathrm{HCHO}$ and $\mathrm{Ag}^{+}$ion in an alkaline solution, the stoichiometric reaction can be written as follows:

$$
\begin{gathered}
2 \mathrm{Ag}^{+}+\mathrm{HCHO}+3 \mathrm{OH}^{-} \rightarrow 2 \mathrm{Ag}+\mathrm{HCOO}^{-}+2 \mathrm{H}_{2} \mathrm{O} \\
2 \mathrm{Ag}^{+}+\mathrm{HCHO}+\mathrm{OH}^{-} \rightarrow 2 \mathrm{Ag}+\mathrm{HCOO}^{-}+\frac{1}{2} \mathrm{H}_{2}
\end{gathered}
$$


The theoretical equations for the production of AgNPs are based on the synthesis procedures in the mixing vessel. The Cannizzaro's reaction in equations (1) and (2) is based on the reduction of $\mathrm{Ag}^{+}$ions during injection of $\mathrm{AgNO}_{3}$ in the primary reacting species of $\mathrm{HCHO}$ and $\mathrm{NaOH}$ to form HCOONa and $\mathrm{CH}_{3} \mathrm{OH}$. Further injection of the metallic $\mathrm{AgNO}_{3}$ into the mixture allows for the reduction of $\mathrm{Ag}^{+}$ion to nanoscale particles. Equations (3) and (4) are based on the addition of $\mathrm{HCHO}$ and then stabilizer to the $\mathrm{AgNO}_{3}$ in the mixing vessel. The nucleophilic addition reaction of $\mathrm{HCHO}$ and the $\mathrm{OH}^{-}$ion occurs, in which the hydride and formate ions are produced. It is the hydride ions that reduce $\mathrm{Ag}^{+}$ion to silver atom and may become hydrogen itself as a by-product.

The development of the above equations has shown that in the course of the reactions, $\mathrm{HCHO}$ is completely converted to other chemical forms that can be more environmentally friendly than HCHO. Zhang et a ${ }^{39}$ reported that during the formation of AgNPs in the solution, there is biodegradation of $\mathrm{HCHO}$ to $\mathrm{HCOO}^{-}$via silver oxidation catalytic activity. ${ }^{40,41}$

\section{Qualitative analysis of AgNPs formation}

The formation of AgNPs from the reacting species was observed by the changes in the color of the solution during the reaction (Figure 1). Factors such as volume of HCHO,

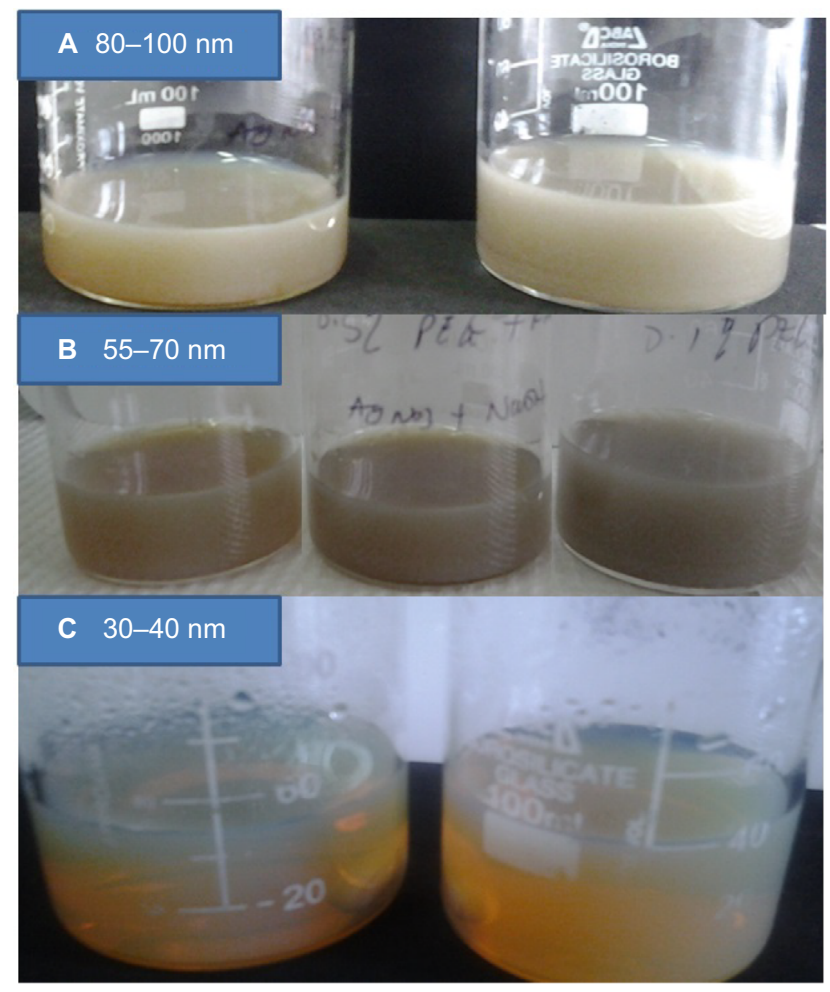

Figure I Reactions of silver nanoparticles at various conditions.

Notes: (A) Shows the retention of color of reacting species which contributed to large particle size above $80 \mathrm{~nm}$, (B) exhibits changes in intensity of color and small particles size and (C) displays the color of silver nanoparticles indicating smallest particle size was formed. holding temperature, reacting time, and concentration of PEG were considered during the reactions to optimize the production. In addition, the sequence of the reacting species including the simultaneous addition, sequential addition, and injection of $\mathrm{AgNO}_{3}$ were considered in several experiments. However, simultaneous addition of reacting species before heating at $80^{\circ} \mathrm{C}$ provided no vivid color changes and maintained the glassy translucent color of PEG. In addition, when $\mathrm{AgNO}_{3}$ and $\mathrm{PEG}$ were mixed and held at a constant heating temperature of $80^{\circ} \mathrm{C}$ with further addition of $\mathrm{HCHO}$ to the mixture, no change of color was observed until some drops of $\mathrm{NaOH}$ solution were added. $\mathrm{NaOH}$ triggered color changes from shiny glassy to slight yellow and then yellow.

When more than $2 \mathrm{~mL}$ of $\mathrm{NaOH}$ solution was added to the reacting species, the color was observed to change from pale yellow to blackish, which indicated the formation of large PS. Similar color changes were observed when a large volume of $\mathrm{HCHO}$ was added to the reacting species containing $\mathrm{AgNO}_{3}$, $\mathrm{PEG}$, and $2 \mathrm{~mL}$ of $\mathrm{NaOH}$. In the injection experiment, when $\mathrm{AgNO}_{3}$ solution was added to a hot solution containing PEG, $\mathrm{HCHO}$, and $\mathrm{NaOH}$, the color was immediately observed to change from glassy to yellow, indicating the formation of AgNPs. However, when the reaction was held at $80^{\circ} \mathrm{C}$ for an extended time, a wool-like blackish color was observed in the solution. Also, increasing the reaction temperature from $80^{\circ} \mathrm{C}$ to $90^{\circ} \mathrm{C}$ accelerated the color change and partition in the solution. ${ }^{42}$ Consequently, qualitative studies have led to the design of new experiments based on the volume and concentration of PEG and reaction time.

The weak reducing agents such as $\mathrm{HCHO}$ do not favor immediate reaction with metallic precursors; they are effective only in solutions of neutral or basic $\mathrm{pH}$ and therefore require a base to complete the reaction. ${ }^{33}$ The addition of alkaline solution such as $\mathrm{NaOH}$ or $\mathrm{Na}_{2} \mathrm{CO}_{3}$ favors higher reducing ability and then color change occurs in the reaction. ${ }^{16,43}$ Increase in $\mathrm{NaOH}$ of the solution increases the $\mathrm{pH}$ of the solution and forms a precipitate at the bottom. This may be caused by diffusion of hydroxyl ion $\left(\mathrm{OH}^{-}\right)$into the stable electric double layer and PEG separation, which attracts the collisions of particles. ${ }^{42}$ When the $\mathrm{pH}$ of solution is increased, the phase separation of PEG occurs, which triggers the precipitation of large particles owing to hydrophobicity of the system in the equilibrium phase. ${ }^{35}$ The dynamic phase separation of PEG may lead to the metal complexation and partition due to chemical participation of solvated anion from the polyethylene oxide chains. ${ }^{6,17,44}$ In addition, at a temperature between $80^{\circ} \mathrm{C}$ and $100^{\circ} \mathrm{C}, \mathrm{NaOH}$ reacts with PEG and precipitate..$^{5,45,46}$ 


\section{DLS}

DLS, also called photon correlation spectroscopy or quasielastic light scattering, is a noninvasive analytical technique for measurement of the size and particle distributions of the particles or molecule at submicron regions. In this study, the DLS was used to characterize the NPs of various formulations and process parameters of AgNPs in laboratory scale. In Figure 2, the reaction time of NPs formation from 1 to $30 \mathrm{~min}$ is shown. The reaction trends show that increasing the reaction time from 1 to $10 \mathrm{~min}$ resulted in a very high NPs nucleation process. However, increase in the reaction time from 10 to $30 \mathrm{~min}$ favored the agglomerations and coalescence of the NPs by forming large PS. This process is attributable to the Ostwald ripening phenomenon, whereby small particles tend to attach to large particles with further effects on the stability and distribution of PS of NPs because of increased solubility and reduced supersaturation of growing species.

It has been reported that reaction time extended beyond 5 min favors particle aggregation at the expense of small PS formed within that time. ${ }^{16}$ Then, the monodispersed nature of the NPs tends to disappear, attracting more inconsistent size distribution of NPs, which leads to unstable and short shelf life of NPs as the cause of aging. ${ }^{16}$ The capping agent tends to lose control of the interfacial mobility, which influences the diffusion of materials and interactions of the reacting species and poor yields due to agglomerations. ${ }^{47}$

PEG concentrations screening for PS formation showed that the increase in PEG concentration from $0.1 \%$ to $1 \%$ weight (wt) decreased the PS of AgNPs from about 100 $\mathrm{nm}$ to about $30 \mathrm{~nm}$. However, increase in PEG concentration from $1 \%$ to $2 \% \mathrm{wt}$ resulted in an increase in PS again (Figure 3). The large NPs were observed to be widely distributed and dispersed and much skewed in DLS analysis. This shows that at around $0.1 \%$ wt PEG, the stability of

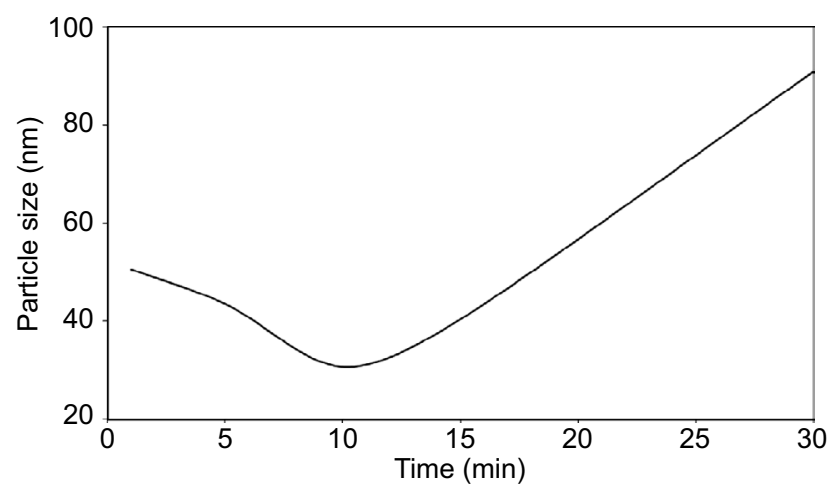

Figure 2 Reaction time.

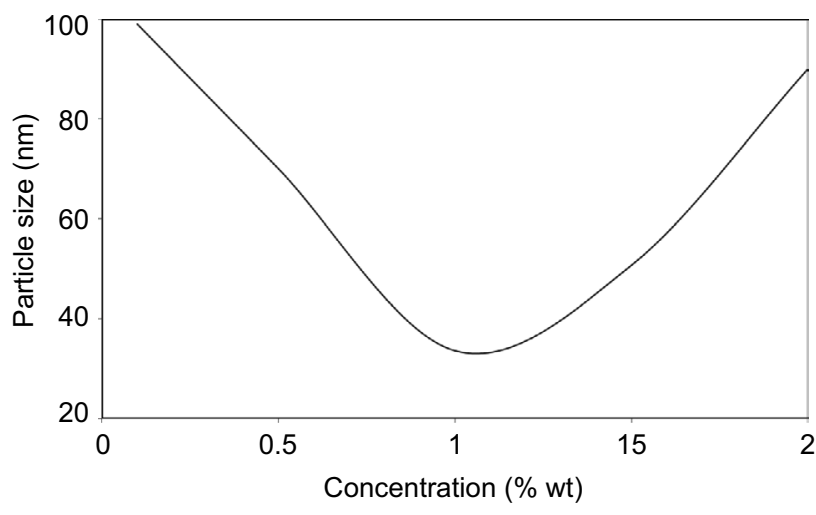

Figure 3 Concentration of PEG.

Abbreviations: PEG, polyethylene glycol; wt, weight.

NPs was low because of high interactions of the reacting species at the interphase as PEG was not able to provide enough separation or coatings to the formed NPs. This leads to aggregation of the NPs into large particles, as shown in the graph. The smallest PS of NPs was observed at $1 \%$ wt PEG and provided enough protection of NPs from agglomeration when stored at room temperature and at $4{ }^{\circ} \mathrm{C}$ for more than 3 mos. At this concentration, PEG provided the maximum stability of NPs as its localization and adsorption at the interphase were predicted to be stronger. ${ }^{8}$ This provides a physical barrier that slows down the agglomeration process, enabling the coating and stabilization process to the formed NPs.

Similarly, the increase in the concentration of PEG above $1 \%$ wt was observed to result in a tremendous increase in the PS. Increase in PEG content in the reacting species served to inhibit the reduction process, which may have led to poor yield of AgNPs owing to the accumulation of AgNPs in the aqueous phase. ${ }^{6,42}$ In addition, the increase in the alkalinity of the solution led to large NPs and precipitation at the bottom of the reacting solution. This may be attributed to the diffusion and collision of the $\mathrm{OH}^{-}$ions in the stable electric double layers.

The contribution of volume to the formation of AgNPs has been observed in similar trends to its concentration. Increase in the volume implies increase in the contents of PEG in the reacting species. Similarly, increasing the volume of PEG solution from 10 to $30 \mathrm{~mL}$ contributed to a decrease in the PS of NPs; however, increasing it to above $25 \mathrm{~mL}$ led to higher PS (Figure 4). Increased PEG content provides higher steric resistance to the diffusion of the NPs at the interphase and provides controlled growth of NPs. However, large NPs at large volumes of PEG may be contributed with poor reaction of $\mathrm{AgNO}_{3}$ in the reacting species and limit the production of the intended NPs. 


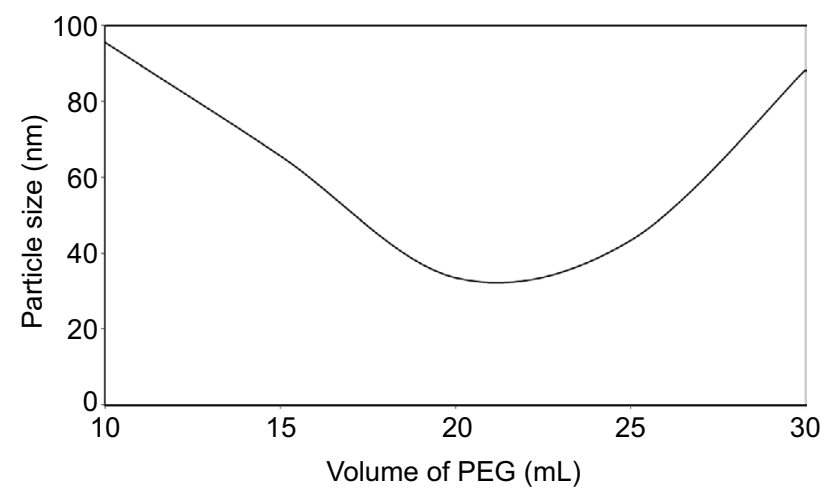

Figure 4 Volume of PEG.

Abbreviation: PEG, polyethylene glycol.

\section{UV-Vis spectrophotometer analysis}

The UV spectra from the UV-Vis spectrophotometer determine the structure of the NPs based on their plasmon oscillations of free surface electrons. ${ }^{48}$ The spectra of AgNPs in Figure 5 exhibit peaks at $450-480 \mathrm{~nm}$. Each peak represents the size and shape of NPs in SPR bands. ${ }^{4,23}$ From SPR bands,

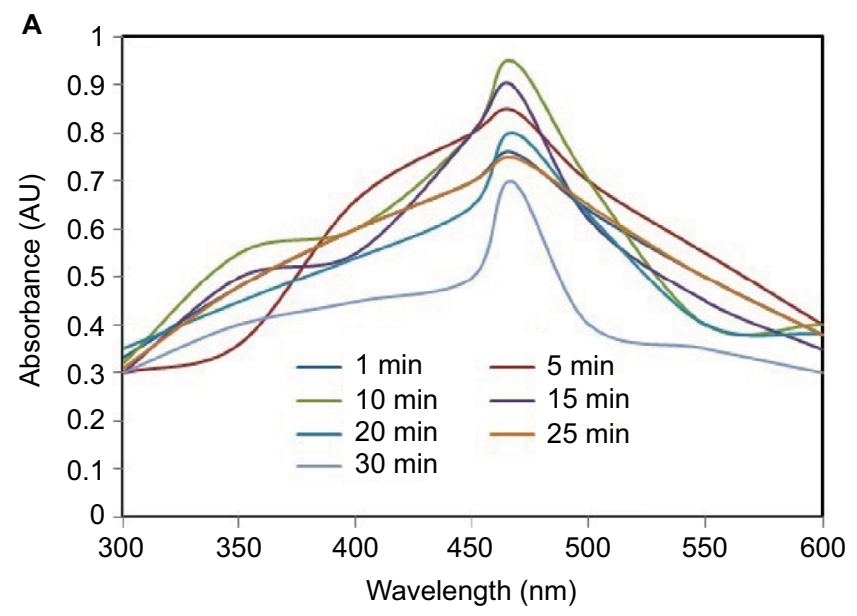

bandwidth increases as the PS increases to $100 \mathrm{~nm}$. The bandwidth of NPs shows their dispersity and the free electron density. The NPs prepared by reaction for $10 \mathrm{~min}$ show the highest peaks in Figure 5A with small peaks at around $320 \mathrm{~nm}$. This is a case for the NPs with some nonspherical particles. The other peaks at this point are caused by the presence of nonspherical particles. The same applies when $20 \mathrm{~mL}$ of PEG was used and 1\% wt PEG. However, for 10 and $15 \mathrm{~mL}$, the particles formed were spherical, as no more than one SPR band is visible.

Similar results have been reported in Raza et al ${ }^{14}$ when $\mathrm{AgNO}_{3}$ was used as metallic precursor, polyvinylpyrrolidone as capping agent, and trisodium citrates and sodium borohydride exhibited spherical AgNPs in the range of 395-510 $\mathrm{nm}$ with triangular NPs at 392 and $789 \mathrm{~nm}$. In this case, Agnihotri et $\mathrm{al}^{6}$ reported that a weak reducing agent such as $\mathrm{HCHO}$ tends to form relatively large NPs of various shapes from triangular, cubic, and rod NPs. Also, Andreescu et a ${ }^{16}$ reported that the plasmon band and its position as a function

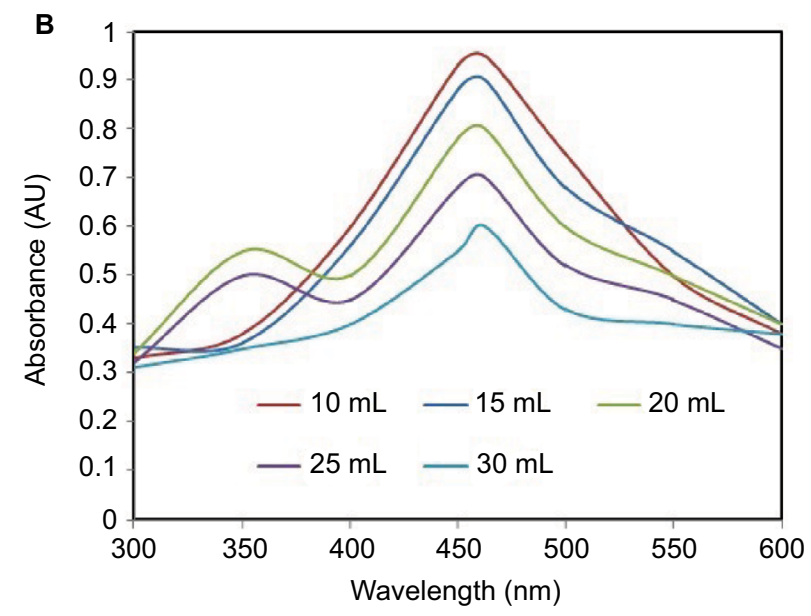

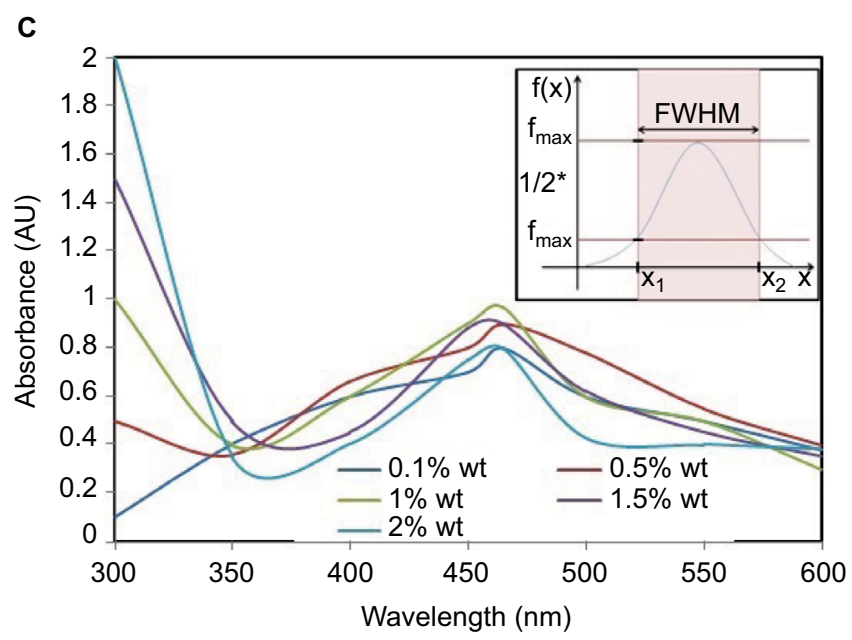

Figure 5 Ultraviolet spectra and FWHM.

Note: (A) Shows the effects of various reaction time, (B) volume of PEG and (C) concentration of PEG on spectra bands of silver nanoparticles.

Abbreviations: FWHM, full width at half maximum; wt, weight; PEG, polyethylene glycol. 
of the reaction time do not change in 3-4 min, but the intensity is increased because of the nucleation process. As the reduction proceeds, the increase in intensity is accompanied by a shift in the position of the peak toward higher wavelength values, indicating an increase in the size of the silver particles because of diffusion growth, aggregation, or a combination of both. ${ }^{13,49}$ Similarly, when the molar ratio of gum Arabic/ silver increased from $0.25: 1.00$ to $0.5: 1.00$ and 1.00:1.00, respectively, there was a drastic reduction in shifting of the plasmon band toward high wavelengths, implying a reduction in PS resulting from an increase in the concentration of the reacting species.

As previously reported, the optical properties of a metallic NP depend mainly on its SPR, where the plasmon refers to the collective oscillation of the free electrons within the metallic NP. ${ }^{6,48}$ The oscillation of electrons in plasmon band depends much on the size, shape, morphology, surfaceadsorbed species, composition, and dielectric environment of the prepared NPs. ${ }^{23,49}$ The SPR of AgNPs tends to shift to longer wavelengths with increasing PS. Pal et $\mathrm{al}^{13}$ reported that only a single SPR band is expected in the absorption spectra of spherical NPs, whereas anisotropic particles could give rise to two or more SPR bands depending on the shape of the particles. Thus, the spherical NPs, disks, and triangular nanoplates of silver show one, two, and more peaks, respectively. ${ }^{23}$

\section{Plasmonic antimicrobial dependence of $\mathrm{AgNPs}$}

The plasmonic antimicrobial effects of AgNPs were determined by inoculation of $E$. coli on the tryptone soya agar plate by surface spreading, as in Figure 6. The antimicrobial studies of the NPs were based on the displayed SPR bands for various formulations, as in "Plasmonic antimicrobial dependence of AgNPs" section. In this case, the selected samples are all with the highest peaks in Figure 5 (A: 10 min reaction time, $\mathrm{B}$ : $10 \mathrm{~mL}$ of $\mathrm{PEG}$ used in reaction, and $\mathrm{C}$ : $1 \%$ wt of PEG used in reaction). The microbial study for the three selected samples showed that the samples formed for $10 \mathrm{~min}$ and with $1 \% \mathrm{wt}$ PEG were less effective against E. coli. The sample prepared using $10 \mathrm{~mL}$ of PEG shows a normal distribution of NPs and was more effective against E. coli. This may be attributed to the monodispersed nature of NPs in the sample, which facilitates the interaction of AgNPs and microbial cells. The 1\% wt PEG showed stronger antimicrobial activity against $E$. coli than the $10 \mathrm{~min}$ reaction time.

In addition, the normally distributed NPs showed narrow bandwidth and higher extinction SPR bands were more

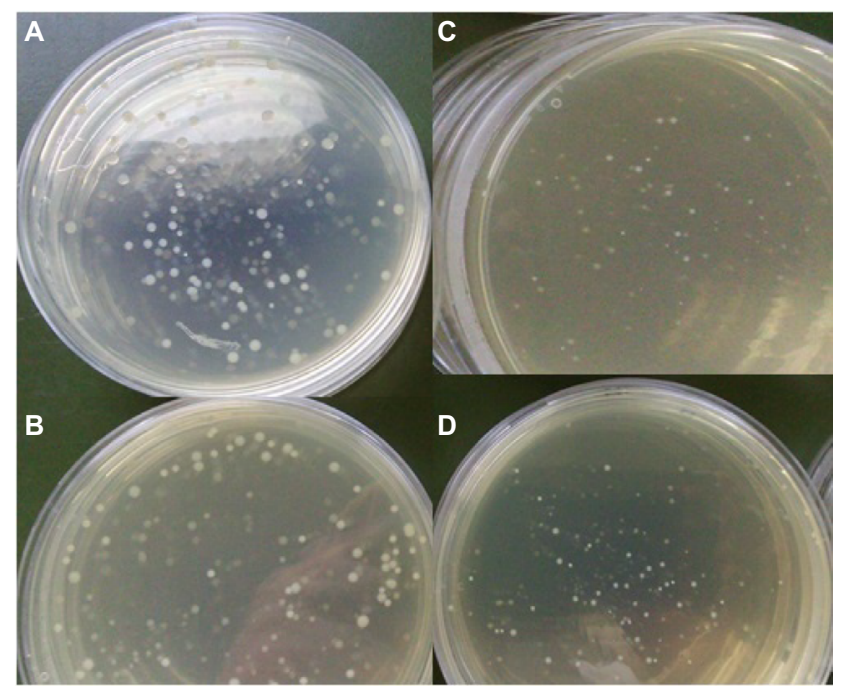

Figure 6 Antimicrobial studies of nanoparticles.

Note: The samples with the highest spectra was tested for antimicrobail activity (A) for 10 min reaction time, (B) from $10 \mathrm{~mL}$ PEG and (C) and (D) for $1 \%$ wt PEG. Abbreviations: PEG, polyethylene glycol; wt, weight.

effective against $E$. coli than other samples. This may be the case where NPs are evenly distributed in regard to size and shape. For broader bandwidth, samples were less effective compared to samples with more than one SPR band. As previously highlighted, samples with more than one peak are anisotropic in nature and have asymmetric orientation. In this case, the contact effectiveness between bacterial cells and NPs is probably reduced. Broader bandwidth NPs have poor size distribution and are polydispersed in nature. Equally, the antimicrobial activities of such NPs would depend on the balance between large PS and small PS particles.

Small and symmetrical NPs exhibiting narrow FWHM have better inhibition against $E$. coli growth compared to the asymmetrical SPR with wider FWHM. These may contribute with low dispersity on NPs due to their larger size distribution compared to narrowly NPs, which are more monodispersed and skewed at the center. The symmetrical NP shows better contact with microbial cells owing to large area-to-volume ratio. In this respect, polydispersed NPs have less contact with microbial cells and thus show insignificant inhibitory effects on $E$. coli growth. Similar findings have been reported by Agnihotri et $\mathrm{al}^{6}$ and Raza et al, ${ }^{14}$ whereby smaller and spherical AgNPs exhibited higher antimicrobial activity for in vitro studies against Pseudomonas aeruginosa and E. coli compared to triangular AgNPs or large spherical PS.

Further observations showed that smaller NPs have higher antimicrobial properties compared to larger NPs because of better contact with microbial cells and their 
tendency to release more $\mathrm{Ag}^{+}$ions that can easily interact with microbial cells compared to larger NPs. ${ }^{2,8,22,23,30,50-52}$ The observations contradict the results of previous research ${ }^{13,24}$ that triangular AgNPs were more effective against $E$. coli than spherical NPs. The report was based on the structural geometry and $\{111\}$ crystal planes being the factors contributing to higher antimicrobial activity of asymmetrical and nonspherical NPs.

\section{Conclusion}

The antimicrobial applications of AgNPs in the food and biomedical industries have increased in recent decades owing to their high spectrum against many strains of spoilage and pathogenic microorganisms in which conventional antimicrobials have become challenging to use. Many studies have analyzed the effects of AgNPs on various microorganisms based on concentrations, sizes, and shapes of the NPs. However, the systems may be disadvantageous in some environments. The uses of features from SPR bands represent the clear and early pictures of assessing the antimicrobial properties of particular particles. This study has found that normally distributed particles with narrow bandwidth show higher antimicrobial properties than NPs with broader bandwidths. The study employed a cheaper and trouble-free method to determine the antimicrobial properties of NPs, avoiding the use of sophisticated instrumentation techniques. However, further research is needed to correlate these results with those obtained from the use of other techniques such as electron microscopy (transmission electron microscopy/ scanning electron microscopy), and X-ray diffraction is needed to confirm the images and the structural planes of the NPs for this study.

\section{Acknowledgments}

The authors appreciate the award of financial support from the Government of Tanzania through the Commission for Sciences and Technology (COSTECH) and the Centre for Science \& Technology of Non-Aligned and Other Developing Countries (NAM S\&T Centre), Government of India, through Research Training Fellowship for Developing Country Scientists (RTF-DCS) 2014/2015. NM thanks the management of the NM-AIST for granting permission to attend the training at the Central Institute of Post-Harvest Engineering and Technology (CIPHET), Ludhiana, Punjab, India, for hosting the research work.

\section{Disclosure}

The authors report no conflicts of interest in this work.

\section{References}

1. Abbasi E, Milani M, Fekri Aval S, et al. Silver nanoparticles: synthesis methods, bio-applications and properties. Crit Rev Microbiol. 2014; 42(2):173-180.

2. Baker C, Pradhan A, Pakstis L, Pochan DJ, Shah SI. Synthesis and antibacterial properties of silver nanoparticles. J Nanosci Nanotechnol. 2005;5(2):244-249.

3. Fayaz AM, Balaji K, Girilal M, Yadav R, Kalaichelvan PT, Venketesan R. Biogenic synthesis of silver nanoparticles and their synergistic effect with antibiotics: a study against gram-positive and gram-negative bacteria. Nanomedicine. 2010;6(1):103-109.

4. Mansouri SS, Ghader S. Experimental study on effect of different parameters on size and shape of triangular silver nanoparticles prepared by a simple and rapid method in aqueous solution. Arab J Chem. 2009;2(1):47-53.

5. Hernández-Castillo MI, Zaca-Moran O, Zaca-Moran P, et al. Shape and stability of silver nanoparticles and their dependence on the conditions of preparation. MRS Proceed. 2012;1371.

6. Agnihotri S, Mukherji S, Mukherji S. Size-controlled silver nanoparticles synthesized over the range 5-100 nm using the same protocol and their antibacterial efficacy. RSC Adv. 2014;4(8):3974-3983.

7. Mlalila N, Kadam DM, Swai H, Hilonga A. Transformation of food packaging from passive to innovative via nanotechnology: concepts and critiques. J Food Sci Technol. 2016;53(9):3395-3407.

8. Chen X, Yan JK, Wu JY. Characterization and antibacterial activity of silver nanoparticles prepared with a fungal exopolysaccharide in water. Food Hydrocol. 2016;53:69-74.

9. Swamy MK, Akhtar MS, Mohanty SK, Sinniah UR. Synthesis and characterization of silver nanoparticles using fruit extract of Momordica cymbalaria and assessment of their in vitro antimicrobial, antioxidant and cytotoxicity activities. Spectrochim Acta A Mol Biomol Spectrosc. 2015;151:939-944.

10. Ramesh PS, Kokila T, Geetha D. Plant mediated green synthesis and antibacterial activity of silver nanoparticles using Emblica officinalis fruit extract. Spectrochim Acta A Mol Biomol Spectrosc. 2015;142:339-343.

11. Pugazhendhi S, Kirubha E, Palanisamy PK, Gopalakrishnan R. Synthesis and characterization of silver nanoparticles from Alpinia calcarata by Green approach and its applications in bactericidal and nonlinear optics. Appl Surf Sci. 2015;357:1801-1808.

12. Ibrahim HMM. Green synthesis and characterization of silver nanoparticles using banana peel extract and their antimicrobial activity against representative microorganisms. J Rad Res Appl Sci. 2015;8(3): 265-275.

13. Pal S, Tak YK, Song JM. Does the antibacterial activity of silver nanoparticles depend on the shape of the nanoparticle? A study of the gram-negative bacterium Escherichia coli. Appl Environ Microbiol. 2007;73(6):1712-1720.

14. Raza M, Kanwal Z, Rauf A, Sabri A, Riaz S, Naseem S. Size- and shapedependent antibacterial studies of silver nanoparticles synthesized by wet chemical routes. Nanomaterials. 2016;6(4):74.

15. Morones JR, Elechiguerra JL, Camacho A, et al. The bactericidal effect of silver nanoparticles. Nanotechnology. 2005;16(10):2346.

16. Andreescu D, Eastman C, Balantrapu K, Goia DV. A simple route for manufacturing highly dispersed silver nanoparticles. J Mater Res. 2007;22(9):2488-2496.

17. Ahmad MB, Lim JJ, Shameli K, Ibrahim NA, Tay MY, Chieng BW. Antibacterial activity of silver bionanocomposites synthesized by chemical reduction route. Chem Cent J. 2012;6(1):101.

18. Badea M, Braic M, Kiss A, et al. Influence of Ag content on the antibacterial properties of $\mathrm{SiC}$ doped hydroxyapatite coatings. Ceram Int. 2016;42(1):1801-1811.

19. Hsueh YH, Lin KS, Ke WJ, et al. The antimicrobial properties of silver nanoparticles in Bacillus subtilis are mediated by released $\mathrm{Ag}^{+}$ions. PLoS One. 2015;10(12):e0144306.

20. Durán N, Durán M, de Jesus MB, Seabra AB, Fávaro WJ, Nakazato G. Silver nanoparticles: a new view on mechanistic aspects on antimicrobial activity. Nanomedicine. 2016;12(3):789-799. 
21. Dhand V, Soumya L, Bharadwaj S, Chakra S, Bhatt D, Sreedhar B. Green synthesis of silver nanoparticles using Coffea arabica seed extract and its antibacterial activity. Mater Sci Eng C Mater Biol Appl. 2016;58:36-43.

22. Guzman M, Dille J, Godet S. Synthesis and antibacterial activity of silver nanoparticles against gram-positive and gram-negative bacteria. Nanomedicine. 2012;8(1):37-45.

23. Martínez-Castañón GA, Niño-Martínez N, Martínez-Gutierrez F, Martínez-Mendoza JR, Ruiz F. Synthesis and antibacterial activity of silver nanoparticles with different sizes. J Nanopart Res. 2008; 10(8):1343-1348.

24. Dong VP, Ha CH, Binh LT, Kasbohm J. Chemical synthesis and antibacterial activity of novel-shaped silver nanoparticles. Int Nano Lett. 2012;2(1):1-9.

25. Dhanalekshmi KI, Meena KS. DNA intercalation studies and antimicrobial activity of $\mathrm{Ag} @ \mathrm{ZrO} 2$ core-shell nanoparticles in vitro. Mater Sci Eng C Mater Biol Appl. 2016;59:1063-1068.

26. Shahverdi AR, Fakhimi A, Shahverdi HR, Minaian S. Synthesis and effect of silver nanoparticles on the antibacterial activity of different antibiotics against Staphylococcus aureus and Escherichia coli. Nanomedicine. 2007;3(2):168-171.

27. Liu Y, Balachandran YL, Li D, Shao Y, Jiang X. PolyvinylpyrrolidonePoly(ethylene glycol) modified silver nanorods can be a safe, noncarrier adjuvant for HIV vaccine. ACS Nano. 2016;10(3):3589-3596.

28. Mohan S, Oluwafemi OS, Songca SP, et al. Synthesis, antibacterial, cytotoxicity and sensing properties of starch-capped silver nanoparticles. J Mol Liq. 2016;213:75-81.

29. Erick ON, Padmanabhan MN. Green chemistry focus on optimization of silver nanoparticles using response surface methodology (RSM) and mosquitocidal activity: Anopheles stephensi (Diptera: Culicidae) Spectrochim Acta A Mol Biomol Spectrosc. 2015;149:978-984.

30. Jeong Y, Lim DW, Choi J. Assessment of size-dependent antimicrobial and cytotoxic properties of silver nanoparticles. Adv Mater Sci Eng. 2014;2014.

31. Xia Y, Xia X, Wang Y, Xie S. Shape-controlled synthesis of metal nanocrystals. MRS Bulletin. 2013;38(4):335-344.

32. Yin H, Yamamoto T, Wada Y, Yanagida S. Large-scale and size-controlled synthesis of silver nanoparticles under microwave irradiation. Mater Chem Phys. 2004;83(1):66-70.

33. Cao G. Zero-dimensional nanostructures: nanoparticles. In: Cao G, Wang Y, editors. Nanostructures and Nanomaterials. Synthesis, Properties and Applications. London, UK: Imperial College Press; 2004:51-109.

34. Chou KS, Ren CY. Synthesis of nanosized silver particles by chemical reduction method. Mater Chem Phys. 2000;64(3):241-246.

35. Chou KS, Lu YC, Lee HH. Effect of alkaline ion on the mechanism and kinetics of chemical reduction of silver. Mater Chem Phys. 2005; 94(2):429-433.

36. Chou KS, Lai YS. Effect of polyvinyl pyrrolidone molecular weights on the formation of nanosized silver colloids. Mater Chem Phys. 2004; 83(1):82-88.
37. Nersisyan HH, Lee JH, Son HT, Won CW, Maeng DY. A new and effective chemical reduction method for preparation of nanosized silver powder and colloid dispersion. Mater Res Bull. 2003;38(6): 949-956.

38. Praus P, Turicová M, Klementová M. Preparation of silver-montmorillonite nanocomposites by reduction with formaldehyde and borohydride. J Braz Chem Soc. 2009;20(7):1351-1357.

39. Zhang J, Li Y, Zhang Y, et al. Effect of support on the activity of Agbased catalysts for formaldehyde oxidation. Sci Rep. 2015;5:12950.

40. Gao L, Gan W, Xiao S, Zhan X, Li J. Enhancement of photo-catalytic degradation of formaldehyde through loading anatase $\mathrm{TiO} 2$ and silver nanoparticle films on wood substrates. RSC Adv. 2015; 5(65):52985-52992.

41. Cui G, Xin Y, Jiang X, et al. Safety profile of TiO2-based photocatalytic nanofabrics for indoor formaldehyde degradation. Int J Mol Sci. 2015;16(11):26055.

42. Zafarani-Moattar MT, Sadeghi R. Phase behavior of aqueous two-phase PEG $+\mathrm{NaOH}$ system at different temperatures. J Chem Eng Data. 2004;49(2):297-300.

43. Gomes JF, Garcia AC, Ferreira EB, et al. New insights into the formation mechanism of $\mathrm{Ag}, \mathrm{Au}$ and $\mathrm{AgAu}$ nanoparticles in aqueous alkaline media: alkoxides from alcohols, aldehydes and ketones as universal reducing agents. Phys Chem Chem Phys. 2015;17(33):21683-21693.

44. Chen J, Spear SK, Huddleston JG, Rogers RD. Polyethylene glycol and solutions of polyethylene glycol as green reaction media. Green Chem. 2005;7(2):64-82.

45. Lee SW, Chang SH, Lai YS, et al. Effect of temperature on the growth of silver nanoparticles using plasmon-mediated method under the irradiation of green LEDs. Materials. 2014;7(12):7781-7798.

46. Luo C, Zhang Y, Zeng X, Zeng Y, Wang Y. The role of poly(ethylene glycol) in the formation of silver nanoparticles. J Colloid Interf Sci. 2005;288(2):444-448.

47. Wei L, Xiao L, He Y. Synthesis of water soluble silver-nanoparticleembedded polymer nanofibers with poly(2-ethyl-2-oxazoline) by a straightforward polyol process. J Mater Res. 2011;26(13):1614-1620.

48. Abou El-Nour KMM, Eftaiha AA, Al-Warthan A, Ammar RAA. Synthesis and applications of silver nanoparticles. Arab J Chem. 2010;3(3):135-140.

49. Balavandy SK, Shameli K, Biak DRBA, Abidin ZZ. Stirring time effect of silver nanoparticles prepared in glutathione mediated by green method. Chem Cent J. 2014;8(1):11.

50. Sotiriou GA, Pratsinis SE. Antibacterial activity of nanosilver ions and particles. Environ Sci Technol. 2010;44(14):5649-5654.

51. Xiu ZM, Zhang QB, Puppala HL, Colvin VL, Alvarez PJJ. Negligible particle-specific antibacterial activity of silver nanoparticles. Nano Lett. 2012;12(8):4271-4275.

52. Meng M, He H, Xiao J, Zhao P, Xie J, Lu Z. Controllable in situ synthesis of silver nanoparticles on multilayered film-coated silk fibers for antibacterial application. J Colloid Interf Sci. 2016;461:369-375.
Nanotechnology, Science and Applications

\section{Publish your work in this journal}

Nanotechnology, Science and Applications is an international, peer-reviewed, open access journal that focuses on the science of nanotechnology in a wide range of industrial and academic applications. It is characterized by the rapid reporting across all sectors, including engineering, optics, bio-medicine, cosmetics, textiles, resource sustainability and science. Applied research into nano-materials, particles, nano-structures and fabrication, diagnostics and analytics, drug delivery and toxicology constitute the primary direction of the journal. The manuscript management system is completely online and includes a very quick and fair peer-review system, which is all easy to use. Visit http://www.dovepress.com testimonials.php to read real quotes from published authors. 